\title{
SYSTEMIC SCLEROSIS AND PULMONARY SARCOIDOSIS, A RARE ASSOCIATION: A CASE REPORT
}

\begin{abstract}
Valéria Bezerra da Silva ${ }^{1}$, Tâmara Melo Santos ${ }^{1}$, Gabriela Almeida Barbosa ${ }^{1}$, Robson Antônio Gonçalves ${ }^{1}$, Maria Roberta Melo Pereira Soares ${ }^{1, \star}$, Sandra Rejane Cabral Batista ${ }^{1}$, Danielle Christinne Soares Egypto ${ }^{1}$, Ana Karla Guedes de Melo ${ }^{1}$, Karla Valéria Miranda de Campos $^{1}$, Alessandra de Sousa Braz ${ }^{1}$, Eutilia Andrade Medeiros Freire ${ }^{1}$
\end{abstract}

1.Universidade Federal da Paraíba, João Pessoa (PB), Brazil.

*Corresponding author: mariarobertam@hotmail.com

\section{BACKGROUND}

Systemic sclerosis (SSc) is a rare disease whose vascular and immunological dysfunction affects organs such as skin, kidneys, lungs and musculoskeletal system. Like SSc, sarcoidosis is also an uncommon systemic disease. The association between these two diseases has been described few times in the literature and, so far, it is unclear whether there is a common link in their etiology.

\section{CASE REPORT}

Patient, 65 years old, retired, hospitalized with a report of pleuritic pain, fever, night sweats, hyporexia and weight loss for six months. Chest tomography was performed, which showed left pleural effusion, mediastinal lymph nodes, multiple centrilobular nodules, better characterized in the upper lobes. The study of the pleural fluid showed exudate with a mononuclear predominance. Pleuroscopy with parietal pleura biopsy was also performed. In view of the findings, treatment for pulmonary tuberculosis (TB) was started. However, at the outpatient return visit, the patient had maintained fever, with worsening of the respiratory pattern and referred to polyarthralgia. The biopsy result showed multiple epithelioid granulomas and sarcoid arrangements, with negative results for TB, fungi and malignancy. The regimen for Koch's bacillus was suspended, and corticosteroid therapy was started, which led to an improvement in pleural effusion and respiratory complaints. During consultation with rheumatology, it was noticed that the patient had findings suggestive of SSc according to the classification criteria for ES proposed by the ACR and EULAR 2013 (skin thickening, digital pulp lesions, Raynaud's phenomenon, telangiectasias, arterial hypertension pulmonary) (Figures 1 and 2). At discharge, maintenance of corticosteroids was advised, as well as the initiation of methotrexate. After two months, the patient
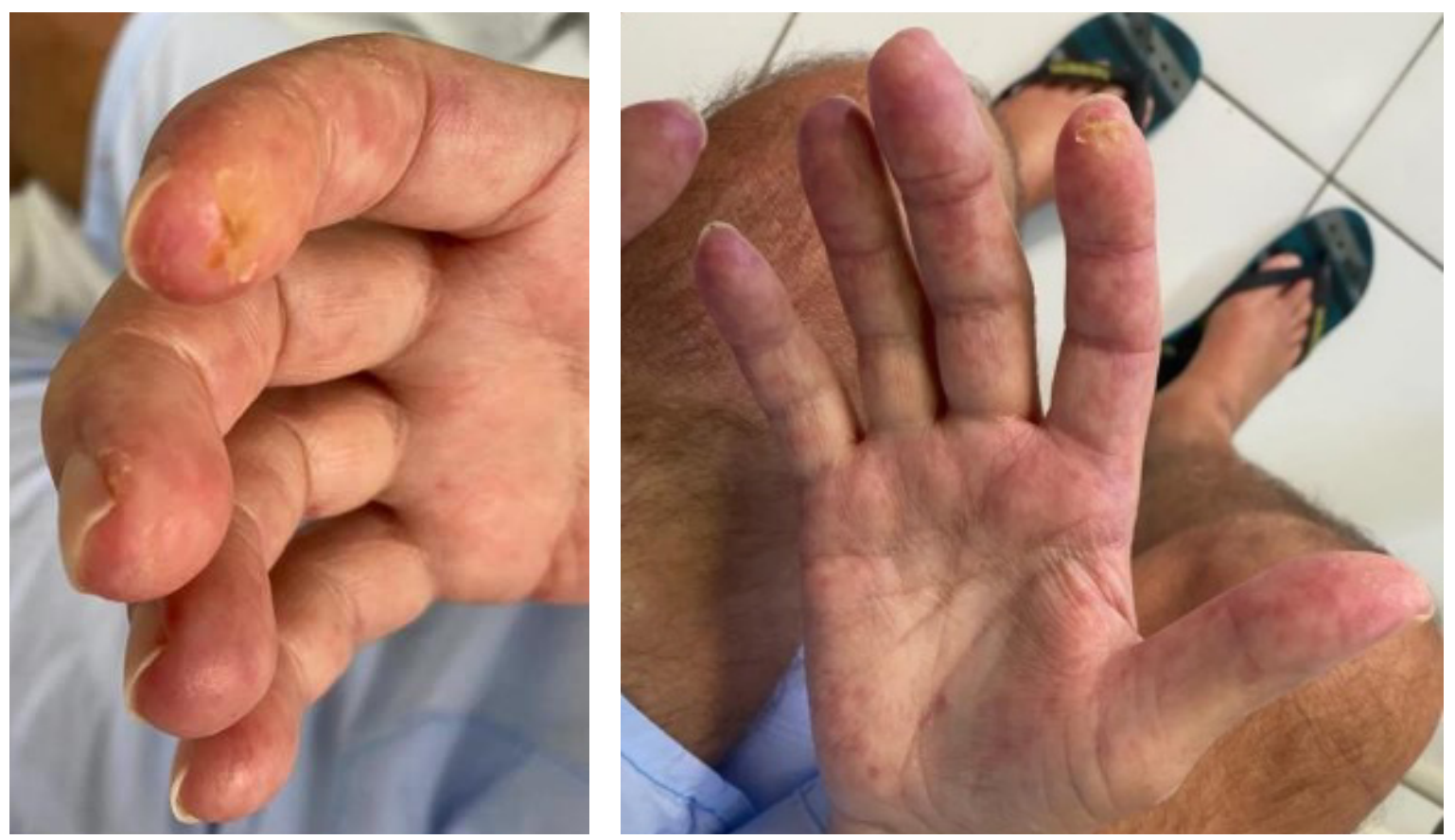

Figure 1. Pitting scars.

Realização: 
was readmitted with dyspnea and increased nitrogenous waste. He needed hemodialysis nine days after the new hospitalization. Captopril was started with the diagnosis of scleroderma renal crisis in mind, however there was no significant improvement beyond the development of hyperkalemia. Renal biopsy corroborated the diagnostic hypothesis of scleroderma renal crisis, bringing as a finding the presence of thrombotic microangiopathy.

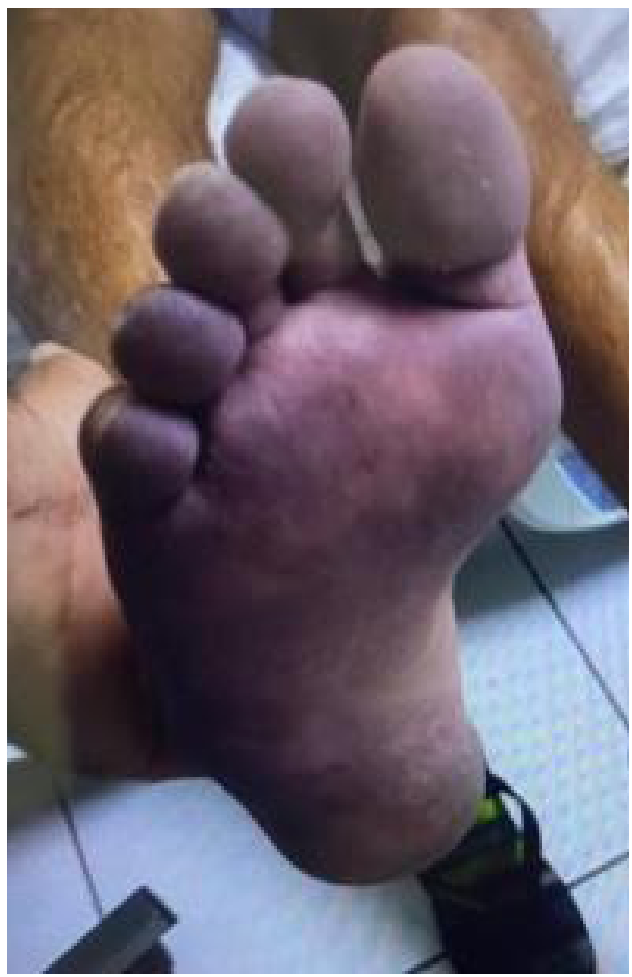

Figure 3. Raynaud's phenomenon.

\section{CONCLUSION}

Even though this is an atypical association, it is important to remember that both diseases can involve the lung, which can lead to confusion in diagnosis and difficulty in management, considering that the treatment of one can worsen some aspects of the other disease.

\section{KEYWORDS}

Systemic sclerosis, Sarcoidosis, Corticosteroids. 